\section{Incidencia de las normas de excepción en la planificación de la verticalización del Área Metropolitana de Santiago}

\author{
Magdalena Vicuña \\ ORCID: https://orcid.org/0000-0002-9476-396X \\ Instituto de Estudios Urbanos y Territoriales, Pontificia \\ Universidad Católica de Chile, Santiago, Chile. Centro \\ Nacional de Investigación para la Gestión Integrada de \\ Desastres Naturales (Cigiden), Santiago, Chile. \\ Correo electrónico: mvicunad@uc.cl
}

\section{Marcela Moraga Zárate \\ ORCID: https://orcid.org/0000-0003-2342-5573 \\ Pontificia Universidad Católica, Santiago, Chile. \\ Universidad Noruega de Ciencia y Tecnología, \\ Trondheim, Noruega.}

\begin{abstract}
Resumen
Este trabajo tiene por objetivo medir cuantitativamente el impacto de la verticalización residencial en la morfología del tejido urbano en barrios seleccionados del Área Metropolitana de Santiago (AMS), a través de la revisión de indicadores de constructibilidad consignados tanto en los permisos de edificación como en los planes reguladores comunales. Complementariamente, se busca comprender en qué medida algunas normas de excepción como premio por fusión predial, conjunto armónico y DFL2 debilitan la capacidad de los planes reguladores comunales para conducir el crecimiento y transformaciones urbanas. Estos beneficios permiten porcentajes adicionales de constructibilidad que sobrepasan lo establecido por los PRC vigentes, y que en suma pueden distanciarse significativamente de la visión de planificación urbana definida para la ciudad. Para el premio por fusión predial corresponde a un máximo de $30 \%$ y para el conjunto armónico oscila entre un 30 y un $50 \%$, según las condiciones del predio a densificar. En tanto, para el beneficio del DFL2 el aumento de constructibilidad considera un máximo de $20 \%$.
\end{abstract}

\section{Palabras clave}

Área Metropolitana de Santiago, constructibilidad, morfología urbana, normas de excepción, planificación urbana, verticalización

\section{Incidence of laws of exception in planning the verticalization of the Metropolitan Area of Santiago}

Financiamiento: Fondecyt $\mathrm{N}^{\circ} 11170501$, Convenio Ministerio de Vivienda y Urbanismo de Chile y Facultad de Arquitectura, Diseño y Estudios Urbanos de la Pontificia Universidad Católica de Chile. Fondap $\mathrm{N}^{\circ} 15110017$.

Agradecimientos: Agradecemos el financiamiento del Convenio Ministerio de Vivienda y Urbanismo de Chile y la Facultad de Arquitectura, Diseño y Estudios Urbanos de la Pontificia Universidad Católica de Chile, y al Proyecto Fondecyt $\mathrm{N}^{\circ} 11170501$ de la Agencia Nacional de Investigación y Desarrollo. Agradecemos también al Centro Nacional de Investigación para la Gestión Integrada de Desastres Naturales (Cigiden), Fondap N ${ }^{\circ} 15110017$, y al Núcleo de Investigación en Gobernanza y Ordenamiento Territorial (NUGOT) del Instituto de Estudios Urbanos y Territoriales UC.

\begin{abstract}
The aim of this work is to measure quantitatively the impact of intensive residential densification over the morphology of the urban fabric in the selected neighborhoods in the Metropolitan Area of Santiago. This is made through the review of different constructability indicators, expressed both in building permits as well as in the respective Communal Urban Plans. Complementarily we seek to understand to what extent some laws of exception such as "plot merging", "harmonic set" and "DFL2", weaken the capacity of communal regulatory plans to lead growth and urban transformations. These benefits allow additional percentages of constructability that exceed what is established by current Urban Plans, which in sum can significatively distance themselves from the urban planning vision defined for the city. The benefit of "plot merging" considers an increase of a maximum of $30 \%$ and for the "harmonic set" it ranges between 30 and $50 \%$, depending on the conditions of the property to be densified. Meanwhile, for the benefit of "DFL2", the increase in constructability considers a maximum of $20 \%$.
\end{abstract}

\section{Keywords}

Constructability, laws of exception, Metropolitan Area of Santiago, urban planning, urban shape, verticalization 


\section{Introducción}

Durante las últimas dos décadas, la verticalización de las ciudades ha adquirido escalas sin precedentes, especialmente en las grandes ciudades del sur global y de Latinoamérica. La verticalización responde principalmente a la densificación residencial, y corresponde a la sustitución del tejido edificado en zonas de alta demanda constructiva y explotación intensa del suelo (De Oliveira et al., 2015). En Chile, la verticalización se ha acrecentado significativamente en sus principales áreas metropolitanas (Livert, \& Gaínza, 2014; Vergara, \& Aguirre, 2019; Vicuña, 2017, 2020) y en aquellas ciudades intermedias en proceso de metropolización. En muchas ocasiones, con un alto costo para los barrios y comunidades que reciben la densificación. En el período 2002-2020, la vivienda en altura concentró un $74 \%$ de los permisos de edificación del Área Metropolitana de Santiago, un 68 \% el Área Metropolitana de Valparaíso y un $42 \%$ en el Área Metropolitana de Concepción, alcanzando para el año 2020 un 94 \%, 83 \% y 69 \%, respectivamente ${ }^{1}$. En efecto, principalmente a partir de la década de 1990, en el Área Metropolitana de Santiago (AMS) ${ }^{2}$ es posible observar procesos de densificación residencial con diversos grados de intensidad en la explotación del suelo y formas de impacto en el entorno urbano (Vicuña, 2020).

Tanto desde el ámbito académico como desde la política pública se ha abordado la problemática de la verticalización, su relación con el mercado inmobiliario (Hidalgo, 2010; Innocenti et al., 2014; López et al., 2012; López-Morales et al., 2015; Vergara Vidal, 2017); incentivos al repoblamiento (Greene et al., 2014); efectos sociales (Contreras, 2011; Inzulza-Contardo, 2012; López-Morales, 2013); e impactos en la habitabilidad (Lopez-Morales et al., 2015; Mora et al., 2013). A pesar de que procesos de densificación residencial balanceados y planificados son promovidos por la agenda internacional (Organización de las Naciones Unidas, 2016) y la política pública (Consejo
Nacional de Desarrollo Urbano [CNDU], 2019), el rol de la planificación urbana en los procesos de verticalización ha sido poco explorado. Esto es especialmente crítico en contextos como el chileno, donde la verticalización tiende a generar externalidades negativas en la habitabilidad urbana y residencial.

El rol de los instrumentos de planificación territorial es fundamental en la conducción de procesos de verticalización que contribuyan a una densificación equilibrada (CNDU, 2019), y a ciudades más inclusivas y equitativas (De Oliveira et al., 2015; Lozano, 1990). Sin embargo, parte importante de las áreas verticalizadas del AMS no han contado con una planificación urbana efectiva. Cuando sí hay normas de altura, densidad y constructibilidad definidas por los planes reguladores comunales (PRC), coexisten formas de regulación centralizadas, que permiten aumentar estos parámetros cuando se trata de proyectos de densificación o de conjuntos de vivienda económica y social (Alcaíno, 2008; Schlack y Vicuña, 2011).

Estos beneficios no representan necesariamente los intereses de las comunidades locales y son aplicados, en particular, en zonas urbanas consolidadas o en procesos de densificación en prácticamente todo el país. Aquí los denominaremos normas de excepción a la planificación, porque constituyen beneficios que se encuentran reglamentados por la Ley General de Urbanismo y Construcciones (LGUC) y su respectiva Ordenanza (OGUC).

Entre estas normas de excepción, se encuentran el premio por fusión predial, el conjunto armónico y el Decreto con Fuerza de Ley $\mathrm{N}^{\circ} 2$. El premio por fusión predial (Artículo 63, LGUC, 2020), se remonta a la década de 1970 cuando el Estado retrocede como actor en el proceso de desarrollo urbano y se establecen incentivos para que desarrolladores privados participen en los procesos

1 Datos elaborados con base en los permisos de edificación, disponibles en el Ministerio de Vivienda y Urbanismo. Ver más en https://www. observatoriourbano.cl/estadisticas-habitacionales/

2 Conforman el Área Metropolitana de Santiago las 34 comunas del núcleo del sistema urbano, correspondiente a la provincia de Santiago y las comunas de San Bernardo y Puente Alto. 
de renovación urbana. Este beneficio se adquiere con la fusión de dos o más terrenos para la realización de una obra nueva y permite incrementar el coeficiente de constructibilidad ${ }^{3}$ en hasta un $30 \%$ respecto del normado por el PRC.

La norma de conjunto armónico (Artículos 107 a 109, LGUC, 2020; Artículos 2.6.4 a 2.6.19, OGUC, 2020) surge como figura legislativa en 1963 como un mecanismo para otorgar mayor flexibilidad en la aplicación de normas en proyectos que aportaran a la ciudad (Alcaíno, 2008). Sin embargo, como plantean Schlack y Vicuña (2011), este se menciona con anterioridad en la Ordenanza Local de Edificación para la Comuna de Santiago (1939), en los PRC de Providencia (1944) y de San Miguel (1951), y en la primera versión de LGUC (1936), siendo su sentido original incentivar la construcción de piezas urbanas significativas (Schlack y Vicuña, 2011). Este beneficio permite incrementar la constructibilidad máxima según determinadas condiciones de dimensión, uso, localización y ampliación del proyecto. De acuerdo con estas condiciones, los proyectos pueden aumentar entre un $30 \%$ y un $50 \%$ la constructibilidad, altura y densidad normada en el PRC.

Por su parte, el Decreto con Fuerza de Ley $\mathrm{N}^{\circ} 2$ (DFL2)

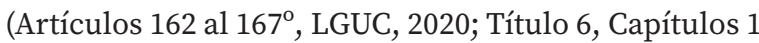
al 5, OGUC, 2020) fue promulgado en 1959 con el objetivo de incentivar la construcción de viviendas económicas en el país. La ley incluye un beneficio tributario para las viviendas de acuerdo con el margen de superficie útil que tengan. En los proyectos DFL2, la superficie común solo se contabiliza para efectos del cálculo de la constructibilidad cuando superan un $20 \%$ de la superficie útil del edificio.

¿Cuánto y cómo han incidido las normas de conjunto armónico, fusión predial y DFL2 en la constructibilidad asociada a los procesos de verticalización del AMS? Este trabajo tiene por objetivo comprender en qué medida dichas normas de excepción debilitan la capacidad de los planes reguladores comunales para conducir el crecimiento por densificación residencial en el AMS, específicamente en lo que respecta a la constructibilidad del proyecto residencial en altura.

\section{Excepcionesa la planificación urbana local}

La planificación urbana es parte de aquellas formas institucionales que constituyen "modos de regulación" y que expresan un determinado régimen (Lipietz, 1986, citado en Harvey, 1998). Para Boyer, la racionalidad tras dichos modos "constituye el origen de las regularidades y reglas dentro del orden económico y (...) no dejan de tener una cierta coherencia y, por lo tanto, una permanencia una vez asegurada su génesis en la historia" (1989, p. 90). Los modos de regulación incorporan ámbitos sociales, económicos y culturales de la sociedad, encarnándose también en la planificación y cristalizando en la morfología urbana (Kostof, 1991).

Existe una tensión entre el carácter fijo y estable que impone la planificación urbana y el movimiento fluido del capital, la cual constituye un problema crucial para la organización social y política del capitalismo (Harvey, 1998), y se refleja en el debate en torno al rol del Estado en la prefiguración de las transformaciones urbanas. Las decisiones que conciernen a la planificación urbana, aunque de corte técnico, son inevitablemente políticas, en tanto asumen valores y justificaciones del poder social (Fainstein, \& Fainstein, 1971). Las limitaciones al derecho de uso y de propiedad y las contradicciones entre el beneficio de quienes acceden a suelo mejor localizado y equipado, y sus efectos e impactos en el bien común (Wai-Chung Lai, 2002), son cuestiones centrales en la planificación urbana.

Sin embargo, tanto en Estados Unidos y Europa, como en Asia y Latinoamérica, con la finalidad de ganar ventajas competitivas en la economía global, los Estados

3 En la Ordenanza General de Urbanismo y Construcciones, este concepto se define como "número que, multiplicado por la superficie total del predio, descontadas de esta última las áreas declaradas de utilidad pública, fija el máximo de metros cuadrados posibles de construir sobre el terreno". 
hacen excepciones a sus prácticas de planificación local (Baptista, 2013). Estas excepciones se materializan en procedimientos especiales para situaciones específicas de gobernanza urbana, que constituyen regímenes de gobierno al margen de la planificación (Baptista, 2013), e implican una "redistribución significativa de poderes, competencias y responsabilidades en la formulación de políticas" (Swyngedouw et al., 2002, p. 556).

En Europa, las medidas de excepcionalidad son crecientemente usadas en la implementación de proyectos urbanos de gran escala (Baptista, 2009; Marrero-Guillamón, 2011; Swyngedouw et al., 2002). Estas medidas extraordinarias permiten examinar cómo la política pública, en lugar de reformar directamente los sistemas de gobernanza y planificación urbana para proyectos particulares, desarrollan arreglos alternativos mediante normas de excepción (Baptista, 2009). La "excepcionalidad" constituiría un rasgo distintivo de la nueva gobernanza urbana, en tanto promueve formas autónomas y dinámicas que relegarían las normas y procedimientos legales de planificación a un segundo plano, basadas en la primacía del "proyecto urbano" (Swyngedouw et al., 2002).

Así, los incentivos a la producción de vivienda en altura, materializados como excepciones a los instrumentos de planificación, permiten analizar el conflicto subyacente a la lógica de las entidades locales que promueven la planificación y las estatales que buscan dar respuesta a la problemática habitacional mediante la promoción de la acción del sector inmobiliario.

\section{Verticalización y planificación urbana (desigual) en el AMS}

La planificación urbana en Chile se realiza a través de instrumentos de escala regional, metropolitana e intercomunal, y comunal. La zonificación de usos de suelo constituye uno de los principales ámbitos de la planificación urbana, y a pesar del estancamiento y retroceso que sufrió en el período de dictadura militar (1973-1990), una década más tarde el Estado impulsó la actualización y creación de instrumentos de planificación territorial (Sanhueza y
Peña-Cortés, 2010), contando así con una mayor cobertura de regulación para el desarrollo urbano (CNDU, 2016).

Las competencias, ámbitos de acción y procesos de elaboración de los instrumentos de planificación territorial se encuentran regulados principalmente en la Ley y la Ordenanza General de Urbanismo y Construcciones.

El Plan Regulador Comunal (PRC) es un instrumento de planificación territorial de carácter normativo, orientado a regular la inversión pública y privada. Es elaborado por los municipios y, a partir de normas urbanísticas, definen usos de suelo, áreas verdes y espacios públicos, así como también establecen límites de constructibilidad, alturas, densidad y ocupación de suelo, entre otras disposiciones. Puede precisar, pero no contradecir, las disposiciones del Plan Regulador Intercomunal (PRI) o Metropolitano (PRM) que afectan el territorio comunal.

Las normas urbanísticas plasmadas en los PRC conllevan, implícita o explícitamente, un modelo de ciudad (Schlack y Vicuña, 2011; Scussel, \& Sattler, 2010) y una idea de urbanidad (Sabaté, 1994; Vicuña, 2015). Consecuentemente, cumplen un rol fundamental en la promoción de una mejor calidad de vida (Orellana et al., 2017), en tanto controlan externalidades negativas de la densificación e incorporan parámetros morfológicos que prefiguran el espacio según un proyecto de ciudad (Diez, 1996).

Ahora bien, la capacidad que tienen los municipios para conducir procesos de verticalización mediante el PRC es disímil. Al disponer de suelo urbano para la explotación intensiva en altura, los municipios compiten entre sí por el acceso al mercado metropolitano de vivienda (López et al., 2012). Mientras algunos demuestran una visión más proclive al mercado de vivienda en altura, otros son más estáticos y responden directamente a los intereses de la comunidad residente (Orellana et al., 2017).

Ante la ausencia de normas actualizadas en el PRC, unos pocos edificios pueden agotar el potencial de crecimiento del tejido urbano (Vicuña, 2017), sin posibilidad de renovarlo en su totalidad, desencadenando una segmentación que se torna irreversible (Diez, 
1996; Vialard, 2012), que incluso puede derivar en tugurización (Innocenti et al., 2014) y precarización (Rojas, 2017). En aquellas comunas con PRC más permisivos o sin PRC, se observa un considerable aumento en la escala de las operaciones inmobiliarias destinadas a vivienda, las que se localizan de forma dispersa en el territorio, superando en algunos casos los 30 pisos y las 4.000 viv/ha (Vicuña, 2020).

El desarrollo inmobiliario en altura ha derivado en que algunas comunas del pericentro adopten normas urbanísticas restrictivas, provocando una segunda ola de "colonización inmobiliaria" hacia sectores que tuvieron un rol industrial-productivo, mediante una alternación del avance inmobiliario y el congelamiento de los PRC (Gerencia de estudios TOCTOC.com, 2020). En el caso de Providencia, una comuna con alto nivel de consolidación de edificaciones en altura, se han adoptado normas aún más restrictivas con el objetivo de regular el uso de las normas de excepción, calculando la constructibilidad máxima en función de los beneficios que el proyecto residencial obtendrá por la fusión predial o la inclusión de departamentos DFL2 (Ilustre Municipalidad de Providencia, 2007).

La verticalización asociada a la densificación residencial no planificada o deficientemente regulada genera efectos negativos en múltiples dimensiones. Desde una dimensión económica, promueve la especulación inmobiliaria (Tella et al., 2011) y la precarización de la vivienda en pos de una mayor rentabilidad (Baptista, 2009). Desde la social, el recambio de habitantes y la expulsión de los propietarios a áreas más periféricas, y la pérdida de sociabilidad dentro de los barrios (Señoret y Link, 2019). Desde una dimensión morfológica espacial, se constata la pérdida y mercantilización de la identidad barrial (Machado y Méndes, 2003), la degradación de condiciones de habitabilidad (López et al., 2015; Rojas, 2017), y la saturación de infraestructuras, servicios y equipamientos, y espacios públicos (Rosa, 2011, citado en De Oliveira et al., 2015; Scussel, \& Sattler, 2010).

Dichos efectos se ven agravados por las normas de excepción a la planificación, que son consecuencia de la descentralización neoliberal, y derivan en una forma de ausencia del Estado en la conducción del desarrollo urbano al asumir una función subsidiaria, para así producir, mantener y gobernar los mercados que este debiera regular (Farías, \& Flores, 2015).

Tal como señalan Laurian et al. (2004), un plan puede considerarse implementado solo en la medida en que incide en la toma de decisiones. Sin embargo, en áreas de alta inversión, el desarrollo urbano tiende a desviarse de los objetivos iniciales de la planificación. En Chile, a pesar de las condicionantes al crecimiento urbano definidas en los PRC, orientadas a plasmar una imagen objetivo, es generalizada la aplicación de normas de excepción que permiten superar las alturas y densidades planificadas. La figura del conjunto armónico es probablemente la más conocida por la publicidad en torno a la judicialización de algunos proyectos, tales como los Conjuntos Armónicos Bellavista (Recoleta) y Portezuelo (Vitacura). En el primer caso, organizaciones sociales se movilizaron para denunciar el impacto irreparable del proyecto al valor patrimonial del sector Bellavista, además de irregularidades en el otorgamiento del permiso de edificación. El proyecto superaba en casi un $100 \%$ la altura normada (Asencio, 2019). En el segundo, los vecinos han apuntado a irregularidades en el proceso de aprobación y a la saturación vehicular que provocarán las 26 torres que contempla el proyecto (Sáez, 2021).

Al no existir una planificación prospectiva que considere el crecimiento en densidad, disponibilidad de infraestructuras y servicios, y variables de integración social, la verticalización provoca una pérdida de confianza entre municipios, inmobiliarias y vecinos (Fedele y Martínez, 2015). Sin embargo, es necesario promover la densificación residencial en áreas subutilizadas y bien localizadas, cautelando la calidad de los barrios densificados.

\section{Metodología}

La selección de los casos de estudio conllevó, en primera instancia, un levantamiento en el AMS mediante fotointerpretación, de clusters de verticalización con torres de más de cinco pisos. Se seleccionaron los sectores con 


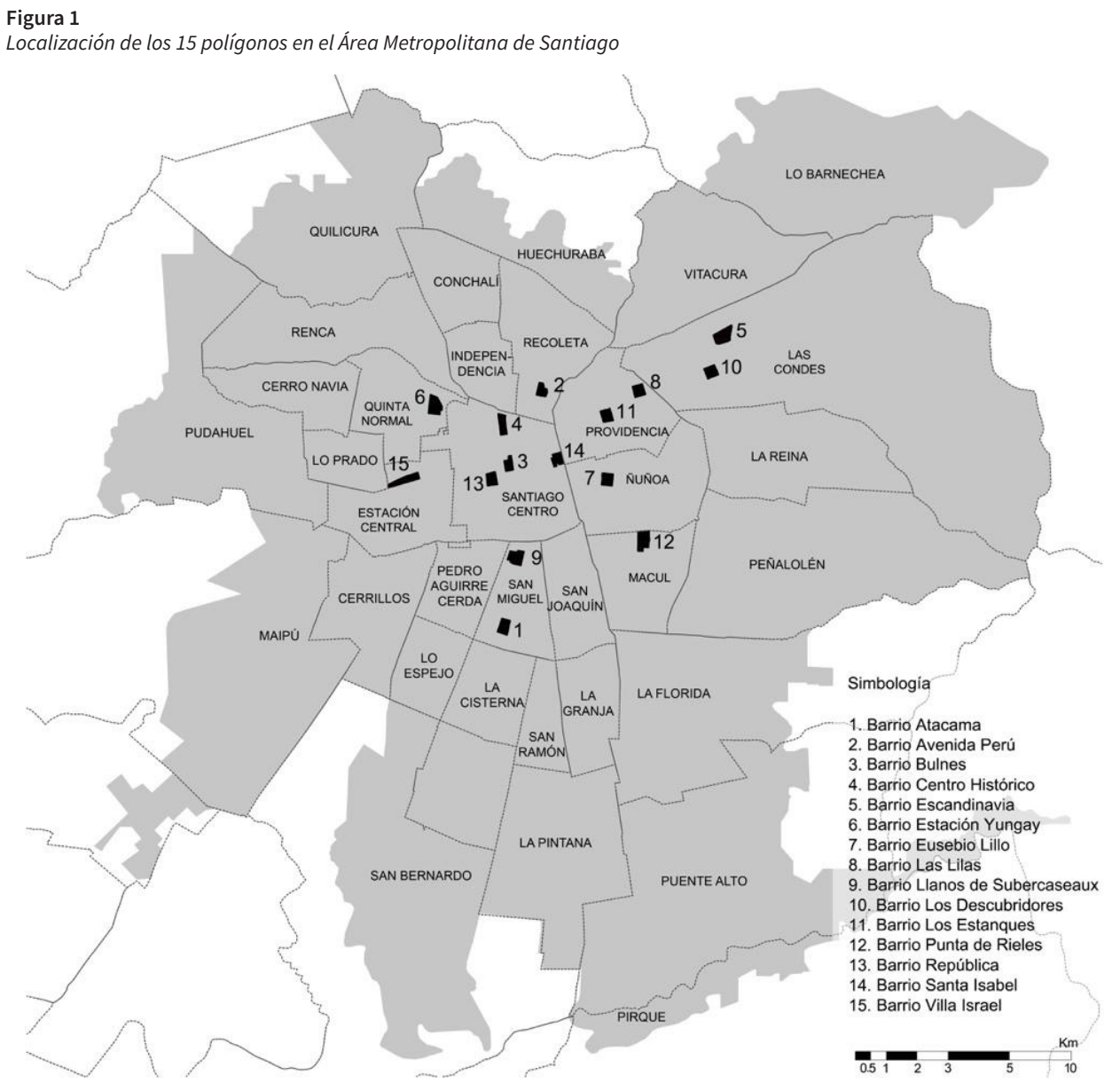

mayor concentración de edificios en altura, realizando un primer filtro de 25 polígonos de aproximadamente 25 hectáreas. Mediante fotointerpretación, estos fueron clasificados en cuatro categorías de estado de avance de la densificación: completa, en densificación, incompleta y segmentada (Diez, 1996). Un segundo filtro permitió la selección de una muestra que representara estos cuatro estados de densificación. Se seleccionaron 15 polígonos, localizados en nueve comunas del $\mathrm{AMS}^{4}$ que mostraran diversidad en términos del estado del proceso de verticalización, intensidad de la densificación, localización y condición socioeconómica (Figura 1). Estas comunas cuentan con PRC con distintos niveles de actualización (entre 1987 y 2018) y, en consecuencia, de especificidad y pertinencia de las normas urbanísticas que proponen.

Se sistematizó la información de los permisos de edificación ${ }^{5}$ correspondientes a 435 torres residenciales localizadas en los 15 polígonos (que denominaremos de aquí en adelante como barrios), las cuales alcanzan una superficie total de 3,8 millones de $\mathrm{m}^{2}$ construidos sobre el nivel del terreno. Se registró la utilización de beneficios de constructibilidad por fusión predial, conjunto armónico y DFL2, siempre y cuando esta información estuviese consignada en el permiso de edificación. A su vez, se revisaron las normas urbanísticas de constructibilidad especificadas en los PRC.

Ahora, el coeficiente de constructibilidad (C.C.) constituye una norma urbanística que representa el número que, multiplicado por la superficie total del predio, fija el máximo de metros cuadrados posibles de construir sobre el terreno. Se calcula a partir de la relación entre la superficie edificada en el terreno sobre el nivel de la calle, y la superficie predial.

4 Estación Central, Quinta Normal, Santiago, San Miguel, Macul, Ñuñoa, Providencia, Las Condes y Recoleta.

5 Permisos de edificación recabados entre agosto y octubre de 2017. 
Los coeficientes de constructibilidad identificados en los permisos de edificación y normados en los PRC son posibles de agrupar en cuatro categorías:

- C.C. proyectado: coeficiente aplicado al proyecto residencial, se encuentra consignado en el permiso de edificación;

- $\quad$ C.C. permitido: coeficiente normado por el PRC al momento de aprobar el permiso de edificación, sin considerar beneficios por fusión predial, conjunto armónico o DFL2. También se encuentra consignado en el permiso de edificación;

- C.C. planificado: coeficiente consignado en la ordenanza del PRC vigente;

- C.C. observado: coeficiente calculado a partir de los datos de superficie total construida sobre el nivel de terreno y superficie del terreno, consignados en el permiso de edificación. Expresa la volumetría real del proyecto residencial en altura porque considera en su cálculo tanto la superficie común como la superficie útil del proyecto residencial en altura.

Posteriormente, se analizaron las diferencias o deltas entre estos cuatro parámetros de constructibilidad. Se sistematizaron cuatro tipos de deltas, los que se ilustran esquemáticamente en la Figura 2 y se describen a continuación:

- Delta impacto real: indica la diferencia entre el C.C. observado, que expresa la volumetría real del edificio, y el C.C. planificado por el PRC vigente. A mayor delta impacto real, mayor es la diferencia entre el C.C. planificado y el C.C. observado. Los deltas equivalentes al $100 \%$ expresan que la constructibilidad observada duplica la planificada por el PRC vigente. Los deltas negativos expresan que el C.C. observado es menor al C.C. planificado.

Este indicador da cuenta de las divergencias entre la constructibilidad materializada y la permitida por los PRC vigentes. Refleja el impacto del edificio residencial en altura en el entorno construido. Puede expresar el uso de beneficios por normas de excepción, o la aplicación de modificaciones al C.C. en el PRC posteriores a la aprobación del proyecto
La muestra analizada para este delta considera los edificios con constructibilidad planificada por el PRC vigente y corresponde a 420 casos. La comuna de Estación Central se excluye de la muestra, ya que no norma coeficiente de constructibilidad.

- Delta fusión predial y/o conjunto armónico: expresa la diferencia entre el C.C. proyectado y el C.C. permitido por el PRC según el permiso de edificación. A mayor delta, mayor es la presencia de beneficios de constructibilidad adicionales al permitido y aplicados al proyecto residencial. Los deltas negativos expresan que el edificio ha empleado un C.C. inferior al permitido por el PRC vigente al momento de aprobación del permiso de edificación. Esto puede explicarse, por ejemplo, por los límites a la altura máxima que impiden ocupar todo el C.C. permitido por el PRC.

Esta muestra considera los permisos de edificación que consignan tanto el C.C. proyectado como el permitido y corresponde a 88 casos. En los barrios de Villa Israel, Estación Yungay, Santa Isabel y Escandinavia no se identificó ningún proyecto con dichos parámetros definidos en el permiso aprobado.

- Delta superficie DFL2: corresponde a la diferencia entre el C.C. observado o real y el C.C. proyectado en el permiso de edificación. A mayor delta, mayor la diferencia entre la constructibilidad consignada como proyectada en el permiso de edificación y la constructibilidad real, indicando, principalmente, mayor beneficio por DFL2. Cuantifica la superficie no contabilizada en el cálculo de la constructibilidad proyectada, pero que sí se materializa sobre el terreno, entendiendo que la constructibilidad realmente aplicada es mayor que la declarada en el permiso.

Esta muestra considera 144 casos e incluye aquellos permisos de edificación que presentan el dato de constructibilidad proyectada. Se identificaron casos dentro de los 15 barrios analizados.

- Delta modificación PRC: expresa la diferencia entre el C.C. consignado como proyectado en el 
Figura 2

Esquema ilustrativo de los cuatro deltas de constructibilidad analizados para los proyectos identificados
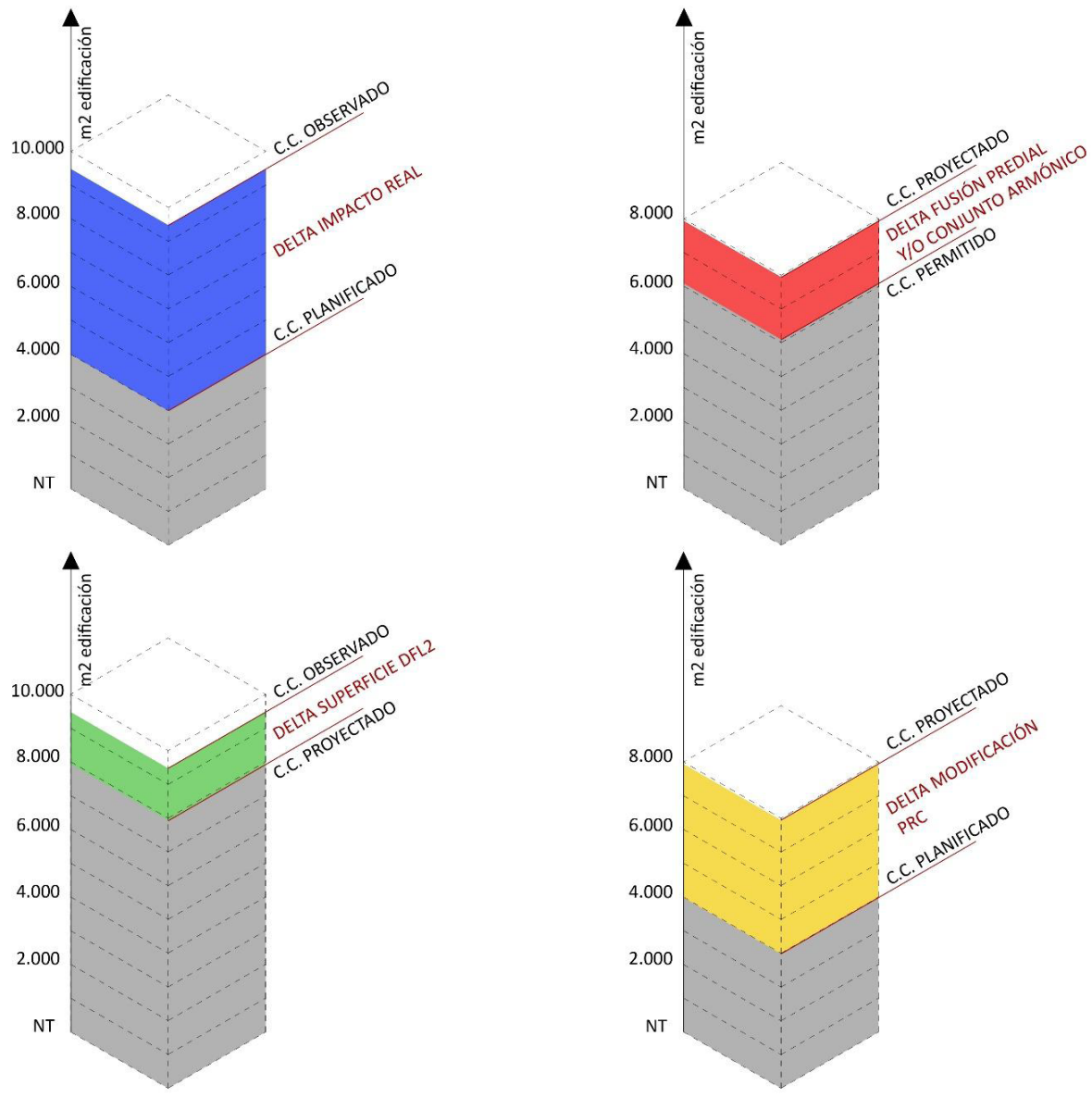

*EJeMPLO ELABORADO EN BASE A EDIFICIO UBICAdO EN BARRIO LLANOS DE SUBERCASEAUX, COMUNA DE SAN MIGUEL, DEL AÑo 2014, CON 19 PISOS DE ALTURA SOBRE NIVEL DE TERRENO (NT).

\begin{tabular}{|lrc|}
\hline \multicolumn{3}{|c|}{ COEFICIENTES DE CONSTRUCTIBILIDAD ANALIZADOS } \\
\hline & C.C. & $\begin{array}{c}\text { SUP. EDIFICABLE } \\
\text { SEGÚN C.C. (M2) }\end{array}$ \\
\hline 1. C.C. PROYECTADO & 2,99 & $7.936,3$ \\
2. C.C. PERMITIDO & 2,30 & $6.104,9$ \\
3. C.C. PLANIFICADO & 1,50 & $3.981,5$ \\
4. C.C. OBSERVADO & 3,57 & $9.471,8$ \\
\hline
\end{tabular}

\begin{tabular}{|lcc|}
\hline \multicolumn{3}{|c|}{ DELTAS DE CONSTRUCTIBIIIDAD ANALIZADOS } \\
\hline & C.C. & $\begin{array}{c}\text { SUP. EDIFICABLE } \\
\text { SEGÚN C.C. (M2) }\end{array}$ \\
\hline $\begin{array}{c}\text { (1) IMPACTO REAL } \\
\text { (C.C. OBSERVADO - C.C. PLANIFICADO) }\end{array}$ & 2,07 & $5.490,4$ \\
$\begin{array}{c}\text { (2) FUSIÓN PREDIAL Y/O CONJUNTO ARMÓNICO } \\
\text { (C.C. PROYECTADO - C.C. PERMITIDO) }\end{array}$ & 0,69 & $1.831,5$ \\
$\begin{array}{c}\text { (3) SUPERFICIE DFL2 } \\
\text { (C.C. OBSERVADO - C.C. PROYECTADO) } \\
\text { (4) MODIFICACION PRC } \\
\text { (C.C. PROYECTADO - C.C. PLANIFICADO) }\end{array}$ & 0,58 & $1.535,6$ \\
\hline
\end{tabular}

permiso de edificación y el C.C. normado por el PRC vigente. A mayor delta, mayor es la brecha entre lo construido y lo planificado actualmente en términos de constructibilidad. Este delta permite visualizar las modificaciones realizadas en los PRC entre el período de edificación de la torre residencial en altura y la planificación vigente. Todo valor positivo refleja una tendencia a limitar la constructibilidad de los edificios en altura. Por el contrario, todo valor negativo indica una modificación en pos de una mayor permisividad normativa.

Los deltas permiten analizar el impacto de las normas de excepción en la planificación de la constructibilidad edificabilidad de la torre. A su vez, dan cuenta de modificaciones a las normas de constructibilidad posteriores a la aprobación de los proyectos, tanto en su tendencia a limitar como a flexibilizar el proceso de 


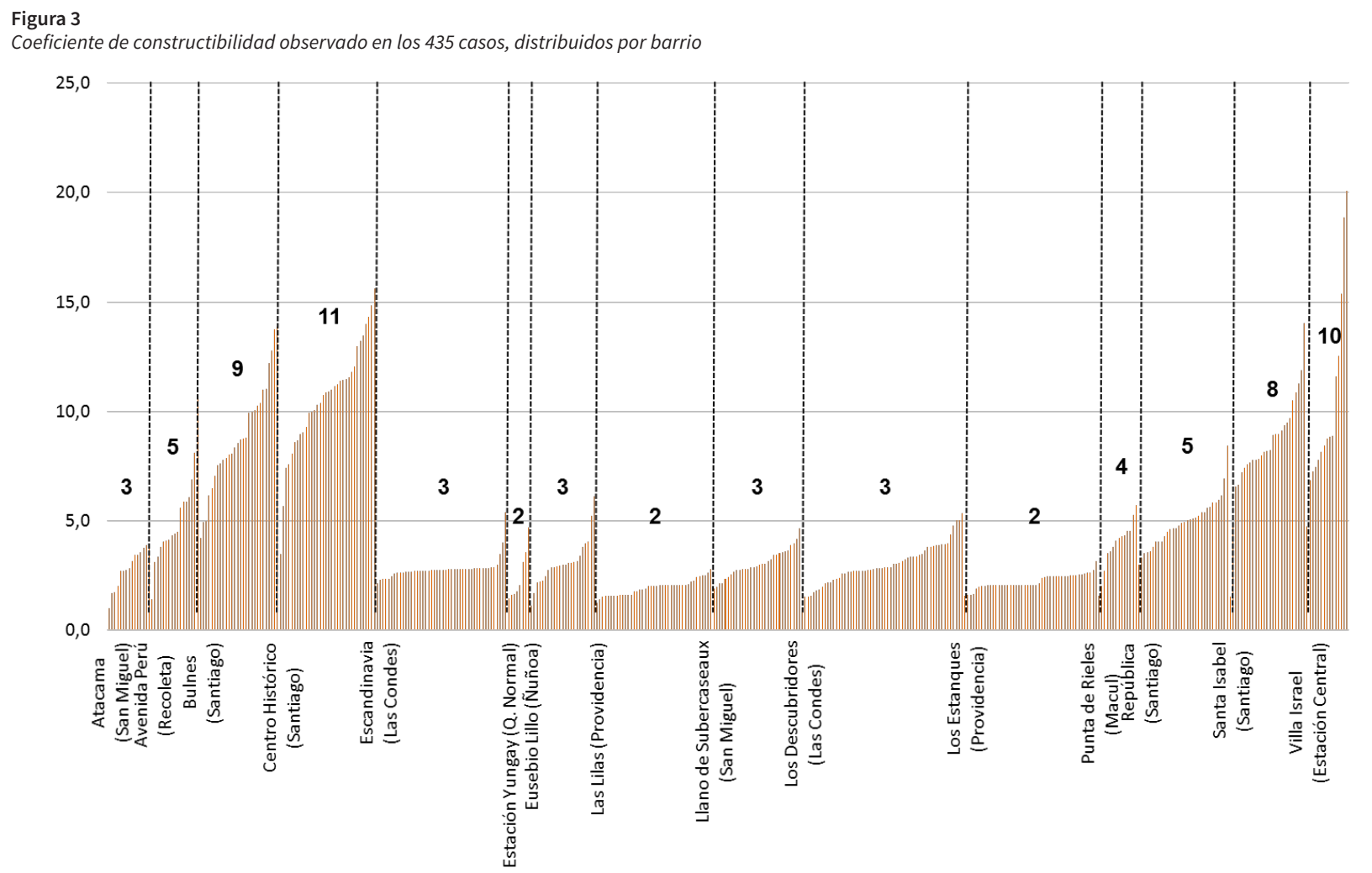

Nota: Gráfico elaborado con base en los permisos de edificación.

densificación, así como las limitaciones que pueden presentar las condiciones de edificabilidad (como alturas máximas o rasantes) para que los proyectos alcancen el C.C. máximo permitido consignado en el permiso de edificación.

\section{Incidencia del premio por fusión predial, conjunto armónico y DFL2 en la constructibilidad del proyecto residencial en altura del AMS}

La utilización de los beneficios de mayor constructibilidad en los 435 casos es disímil. Mientras prácticamente todos los casos analizados $(99,8 \%)$ se acogen al beneficio de DFL2, un $63 \%$ obtuvo el premio por fusión predial, y apenas un 0,2 \% se acogió a los beneficios de conjunto armónico.

Los coeficientes de constructibilidad alcanzados por los casos varían ampliamente (Tabla 1). Los casos promedian un C.C. observado de 4,61, con una desviación estándar alta, correspondiente a 3,32. A nivel de polígonos, el promedio de constructibilidad más bajo corresponde a 2 (Figura 3). El promedio más alto corresponde a 11, en el Centro Histórico. Mientras en Las Lilas (Providencia) la desviación estándar del C.C. corresponde a 1,95, en el Centro Histórico es de 10,37. Los barrios que presentan una desviación estándar superior a la media y que, por lo tanto, poseen un alto grado de heterogeneidad en los parámetros de constructibilidad son: Punta de Rieles, República, Avenida Perú, Santa Isabel y Villa Israel. Dichas diferencias dan cuenta de la diversidad de los procesos de verticalización en términos de la fragmentación del tejido urbano (Vicuña, 2017). En la escala del edificio, la constructibilidad más alta registrada corresponde a 20,1 (Villa Israel), y la más baja corresponde a 1,0 (Atacama).

La Figura 4 presenta el desempeño de las curvas correspondientes a cada uno de los deltas. Mientras los deltas impacto real y modificaciones PRC muestran mayor variabilidad, los deltas beneficios de excepción y superficie DFL2 presentan una variabilidad por debajo del $30 \%$. En Villa Israel, Las Lilas, Atacama, Punta de Rieles, Eusebio Lillo, Los Estanques y, en cierta medida, Escandinavia, los porcentajes de delta son menores. En Estación Yungay y Bulnes los deltas son mayores, sobrepasando el $100 \%$. A continuación, analizaremos el desempeño de cada uno de los deltas de constructibilidad propuestos. 
Tabla 1

Síntesis de datos promedio obtenidos por barrio de cuatro deltas analizados

\begin{tabular}{|c|c|c|c|c|c|c|c|c|c|}
\hline \multirow[t]{2}{*}{ Barrio } & \multirow[t]{2}{*}{ Comuna } & \multicolumn{2}{|c|}{$\begin{array}{l}\text { Delta impacto real } \\
\text { C.C. observado (PE) - C.C. } \\
\text { planificado (PRC) }\end{array}$} & \multicolumn{2}{|c|}{$\begin{array}{l}\text { Delta fusión predial y } \\
\text { conjunto armónico } \\
\text { C.C. proyectado (PE) - C.C. } \\
\text { permitido (PE) }\end{array}$} & \multicolumn{2}{|c|}{$\begin{array}{l}\text { Delta superficie DFL2 } \\
\text { C.C. observado (PE) - C.C. } \\
\text { proyectado (PE) }\end{array}$} & \multicolumn{2}{|c|}{$\begin{array}{l}\text { Delta modificaciones PRC } \\
\text { C.C. proyectado (PE) - C.C } \\
\text { planificado (PRC) }\end{array}$} \\
\hline & & $\begin{array}{l}\text { Promedio } \\
\text { neto }\end{array}$ & $\begin{array}{l}\text { Promedio } \\
\text { porcentaje }\end{array}$ & $\begin{array}{l}\text { Promedio } \\
\text { neto }\end{array}$ & $\begin{array}{l}\text { Promedio } \\
\text { porcentaje }\end{array}$ & $\begin{array}{l}\text { Promedio } \\
\text { neto }\end{array}$ & $\begin{array}{l}\text { Promedio } \\
\text { porcentaje }\end{array}$ & $\begin{array}{l}\text { Promedio } \\
\text { neto }\end{array}$ & $\begin{array}{l}\text { Promedio } \\
\text { porcentaje }\end{array}$ \\
\hline Atacama & San Miguel & 0,01 & $2,50 \%$ & 0,2 & $9,30 \%$ & 0,47 & $15,76 \%$ & 0,2 & $9,35 \%$ \\
\hline Avenida Perú & Recoleta & 2,8 & $127,24 \%$ & 0,8 & $34,80 \%$ & 0,68 & $20,33 \%$ & 0 & $-0,15 \%$ \\
\hline Bulnes & Santiago & 5,06 & $135,24 \%$ & 0 & $-0,50 \%$ & 0,71 & $9,97 \%$ & 5,2 & $143,07 \%$ \\
\hline Centro Histórico & Santiago & 4,05 & $66,38 \%$ & $-0,6$ & $-7,50 \%$ & 1,62 & $18,45 \%$ & 3,1 & $47,71 \%$ \\
\hline Escandinavia & Las Condes & 0,96 & $51,50 \%$ & 0,5 & $29,00 \%$ & 0,42 & $17,61 \%$ & 0,5 & $29,02 \%$ \\
\hline Estación Yungay & Quinta Normal & 1,29 & $107,82 \%$ & - & - & 0,11 & $3,40 \%$ & 2 & $169,17 \%$ \\
\hline Eusebio Lillo & Ñuñoa & 0,55 & $26,42 \%$ & 0,5 & $22,40 \%$ & 0,6 & $19,68 \%$ & $-0,1$ & $2,17 \%$ \\
\hline Las Lilas & Providencia & 0,24 & $14,01 \%$ & 0,5 & $30,00 \%$ & 0,18 & $9,15 \%$ & 0,2 & $9,17 \%$ \\
\hline $\begin{array}{l}\text { Llano de } \\
\text { Subercaseaux }\end{array}$ & San Miguel & 0,75 & $33,84 \%$ & 0,3 & $11,90 \%$ & 0,52 & $18,36 \%$ & 0,3 & $11,92 \%$ \\
\hline Los Descubridores & Las Condes & 0,96 & $51,52 \%$ & 0,4 & $27,50 \%$ & 0,43 & $16,46 \%$ & 0,8 & $44,88 \%$ \\
\hline Los Estanques & Providencia & 0,46 & $26,17 \%$ & 0,3 & $18,00 \%$ & 0,38 & $17,80 \%$ & 0,3 & $16,90 \%$ \\
\hline Punta de Rieles & Macul & 0,32 & $7,87 \%$ & 0,14 & $5,70 \%$ & 0,75 & $20,28 \%$ & $-0,3$ & $-6,51 \%$ \\
\hline República & Santiago & 0,72 & $23,78 \%$ & 0,73 & $20,40 \%$ & 0,65 & $15,27 \%$ & 0,11 & $5,68 \%$ \\
\hline Santa Isabel & Santiago & 2,82 & $51,10 \%$ & - & - & 1,37 & $16,17 \%$ & 2,8 & $50,53 \%$ \\
\hline Villa Israel & $\begin{array}{l}\text { Estación } \\
\text { Central }\end{array}$ & - & - & - & - & 1,61 & 0,1762 & - & - \\
\hline \multicolumn{2}{|c|}{ Promedio edificios analizados } & 1,4 & $46,57 \%$ & 0,45 & $22,10 \%$ & 0,66 & $16,95 \%$ & 0,57 & $25,25 \%$ \\
\hline
\end{tabular}

Nota: Tabla elaborada con base en los permisos de edificación.

Figura 4

Desempeño promedio de cuatro deltas analizados, por barrio y en porcentaje

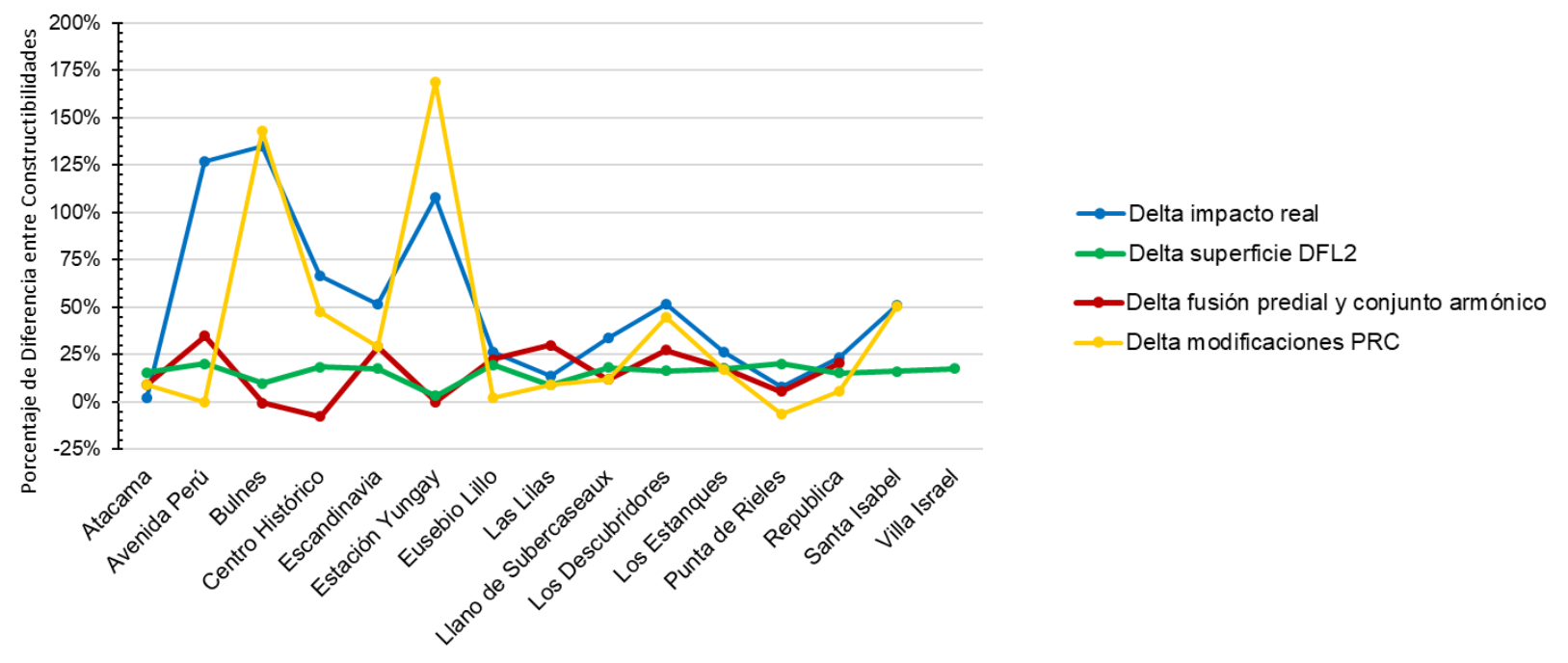


Figura 5

Coeficiente de constructibilidad observado en los 435 casos, distribuidos por barrio

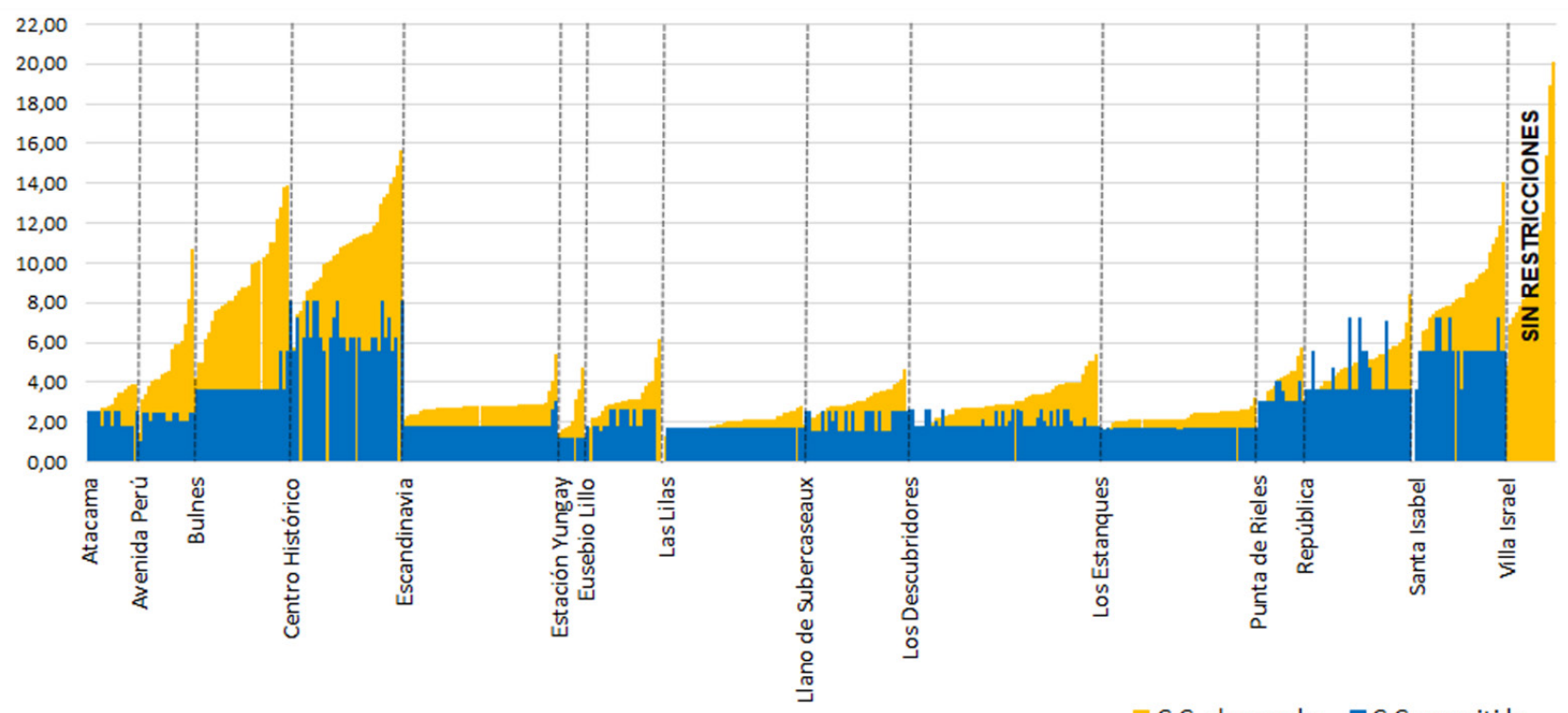

C.C. observado $\square$ C.C. permitido

\section{Delta impacto real: supremacía de la constructibilidad observada por sobre la planificada}

Este delta alcanza un promedio de 46,6 \% (Tabla 1), el cual corresponde a un C.C. neto de 1,4, equivalente a la diferencia entre el C.C. promedio observado $(4,6)$ y el C.C. planificado $(3,2)$ (Figura 2). En total, este delta equivale a $1.123 .666 \mathrm{~m}^{2}$ edificados y en 12 de los 15 barrios corresponde al más alto de los deltas. También es el que presenta mayor variabilidad: mientras en Bulnes alcanza un 135,2 \%, en Atacama apenas representa un 2,5\%.

Es posible identificar barrios con un alto grado de variabilidad en términos de la constructibilidad observada en los proyectos residenciales. Tal es el caso de Avenida Perú, Bulnes, Centro Histórico, Santa Isabel y Villa Israel ${ }^{6}$ (Figura 5). Sin embargo, el C.C. planificado, por lo general inferior al C.C. observado, tiende a presentar pocas variaciones a nivel de barrio. Las Lilas y Los Estanques, en Providencia, presentan el delta impacto real más bajo porque ambos coeficientes son similares. Contrariamente, Avenida Perú, Bulnes, Centro Histórico y Santa Isabel presentan la mayor distancia entre ambos coeficientes, pudiendo incluso duplicarse el C.C. observado respecto del C.C. planificado.
Existe una mayor concentración de delta impacto real, es decir, mayor brecha entre constructibilidad observada y actualmente planificada, en los períodos 2001-2005 y 2006-2010, con un promedio de delta correspondiente a 1,5 y 2, respectivamente (Tabla 2). En comunas como Santiago y Recoleta, por ejemplo, los PRC han disminuido significativamente la constructibilidad permitida desde la aprobación de los permisos de edificación analizados. El promedio alcanzado por los 14 barrios asciende a $83.567 \mathrm{~m}^{2}$ de delta impacto real. Esto es especialmente crítico en el Barrio Bulnes, donde dicho delta representa una diferencia del 135,24 \% entre el C.C. observado y el planificado. Este delta equivale a una superficie adicional de 223.790,6 $\mathrm{m}^{2}$ con respecto a la constructibilidad permitida, la cual equivale aproximadamente a 32 edificios de 15 pisos.

La variación del delta impacto real en la escala barrial responde a dos factores principales. Primero, al alto uso de beneficios que permiten alcanzar constructibilidades superiores a las establecidas en el PRC, como el DFL2, pero, en particular, por el uso del premio por fusión predial. En los barrios Centro Histórico, Santa Isabel, Escandinavia, Avenida Perú, Bulnes y Los Descubridores, dicho beneficio se utiliza en al menos el $50 \%$ de los

6 Presentan las desviaciones estándar de C.C. más altas de la muestra: 2,1 en Avenida Perú; 2,49 en Bulnes; 2,54 en Centro Histórico; 2,32 en Santa Isabel; y 4,48 en Villa Israel. 
Tabla 2

Delta impacto real según barrio y periodo temporal, entre 1990 y 2017 para 14 barrios del AMS

\begin{tabular}{|c|c|c|c|c|c|c|c|c|c|c|c|c|}
\hline \multirow{2}{*}{ BARRIO } & \multicolumn{2}{|c|}{ 1990-1995 } & \multicolumn{2}{|c|}{$1996-2000$} & \multicolumn{2}{|c|}{ 2001-2005 } & \multicolumn{2}{|c|}{ 2006-2010 } & \multicolumn{2}{|c|}{$2011-2017$} & \multicolumn{2}{|r|}{ Total } \\
\hline & C.C & Porcentaje & C.C & Porcentaje & C.C & Porcentaje & C.C & Porcentaje & C.C & Porcentaje & C.C & Porcentaje \\
\hline Atacama & $-2,2$ & $-69 \%$ & $-0,8$ & $-25 \%$ & $-0,4$ & $-15 \%$ & 0,5 & $17 \%$ & 1,1 & $42 \%$ & 0,0 & $3 \%$ \\
\hline Avenida Perú & 1,7 & $73 \%$ & 3,1 & $159 \%$ & 3,4 & $152 \%$ & 1,7 & $70 \%$ & 0,3 & $10 \%$ & 2,8 & $127 \%$ \\
\hline Bulnes & - & $0 \%$ & 4,4 & $122 \%$ & 6,2 & $159 \%$ & 5,4 & $145 \%$ & 2,9 & $80 \%$ & 5,1 & $135 \%$ \\
\hline Centro Histórico & - & $0 \%$ & 1,0 & $12 \%$ & 3,1 & $55 \%$ & 6,2 & $99 \%$ & 0,7 & $9 \%$ & 4,2 & $71 \%$ \\
\hline Escandinavia & 1,1 & $59 \%$ & 0,7 & $38 \%$ & 0,9 & $51 \%$ & 1,1 & $54 \%$ & 1,1 & $60 \%$ & 1,0 & $52 \%$ \\
\hline Estación Yungay & - & $0 \%$ & - & $0 \%$ & 0,3 & $22 \%$ & 1,2 & $92 \%$ & 3,5 & $291 \%$ & 1,3 & $108 \%$ \\
\hline Eusebio Lillo & 0,5 & $30 \%$ & 0,7 & $38 \%$ & 0,5 & $28 \%$ & 0,6 & $24 \%$ & - & $0 \%$ & 0,6 & $26,93 \%$ \\
\hline Las Lilas & 0,2 & $12 \%$ & 0,0 & $2 \%$ & 0,4 & $23 \%$ & 0,5 & $30 \%$ & - & $0 \%$ & 0,2 & $13 \%$ \\
\hline $\begin{array}{l}\text { Llano de } \\
\text { Subercaseaux }\end{array}$ & 0,9 & $54 \%$ & 0,6 & $23 \%$ & 0,8 & $40 \%$ & 0,8 & $28 \%$ & 0,8 & $36 \%$ & 0,8 & $39 \%$ \\
\hline Los Descubridores & 1,6 & $89 \%$ & 0,8 & $38 \%$ & 1,1 & $55 \%$ & - & $0 \%$ & 0,4 & $19 \%$ & 1,2 & $64 \%$ \\
\hline Los Estanques & 0,3 & $19 \%$ & 0,3 & $20 \%$ & 0,7 & $39 \%$ & 0,8 & $47 \%$ & 0,3 & $15 \%$ & 0,5 & $27 \%$ \\
\hline Punta de Rieles & $-1,4$ & $-47 \%$ & - & $0 \%$ & 0,3 & $10 \%$ & 0,6 & $14 \%$ & 0,4 & $10 \%$ & 0,3 & $8 \%$ \\
\hline República & 1,0 & $34 \%$ & 1,0 & $32 \%$ & 1,3 & $35 \%$ & 0,4 & $17 \%$ & 0,7 & $20 \%$ & 0,9 & $28 \%$ \\
\hline Santa Isabel & - & $0 \%$ & 1,3 & $24 \%$ & 3,1 & $54 \%$ & 3,8 & $71 \%$ & 1,2 & $21 \%$ & 3,0 & $55 \%$ \\
\hline Total general & 0,8 & $42 \%$ & 1,2 & $44 \%$ & 1,6 & $55 \%$ & 2,6 & $65 \%$ & 1,0 & $38 \%$ & 1,6 & $52 \%$ \\
\hline
\end{tabular}

casos. Los valores más bajos del delta impacto real se concentran en barrios que cuentan con indicaciones en el PRC para la aplicabilidad de las normas de excepción, como Atacama, Punta de Rieles, Eusebio Lillo, Los Estanques y Las Lilas. A pesar de contar con un mayor nivel de restricción en el uso de este beneficio, no corresponden necesariamente a los barrios con menor presencia del premio por fusión predial.

En segundo lugar, la variabilidad del delta impacto real responde a modificaciones de la norma de C.C. posteriores a la aprobación de los permisos de edificación, las cuales buscan restringir la verticalización. En Estación Yungay, el PRC de 2002 no consideraba C.C, pero sí una altura máxima de 18 metros, que se redujo a 10,5 metros en 2016, con un C.C de 1,2. Por su parte, en Santa Isabel el C.C. se modificó en 2011, reduciéndose de un máximo de 7,2 a 4,8. En Bulnes, en 2011 se redujo la altura máxima de 35 a 22,5 metros con un C.C. de 3,6.
Ahora bien, si analizamos las diferencias entre C.C. observado y C.C. planificado en la escala del edificio, podemos constatar que los deltas impacto real son aún mayores (Figura 6). Observamos que si bien los barrios pertenecientes a Las Condes y Providencia, y El Llano de Subercaseaux en San Miguel intensifican sus procesos de densificación en el período 1991-1995, los deltas obtenidos en la mayor parte de estos períodos tienden a ser menores a $100 \%$, no distanciándose significativamente de la norma actualmente vigente ${ }^{7}$. Algo similar observamos en comunas como Recoleta, Ñuñoa y Santiago en los períodos 1995-2000 y 2001-2005.

Entre 2004 y $2009^{8}$, se produce un fenómeno de intensificación de la constructibilidad de los proyectos residenciales en altura, con deltas impacto real altos, especialmente en los barrios Santa Isabel, Centro Histórico y Bulnes. Desde el año 2010, el proceso de densificación residencial fue avanzando, aunque con menor intensidad que en los períodos anteriores y con deltas de "impacto real" más bajos.

7 A excepción de algunos edificios en los barrios Avenida Perú y Bulnes, en los cuales se presentan mayoritariamente deltas superiores a $100 \%$ e incluso algunos que sobrepasan el $200 \%$, con constructibilidades observadas mayores que 4,5 y 7,5, respectivamente.

8 En función de los resultados obtenidos y presentados en el gráfico, para este análisis se identifican rangos que difieren de la clasificación anterior ordenada en lustros. 


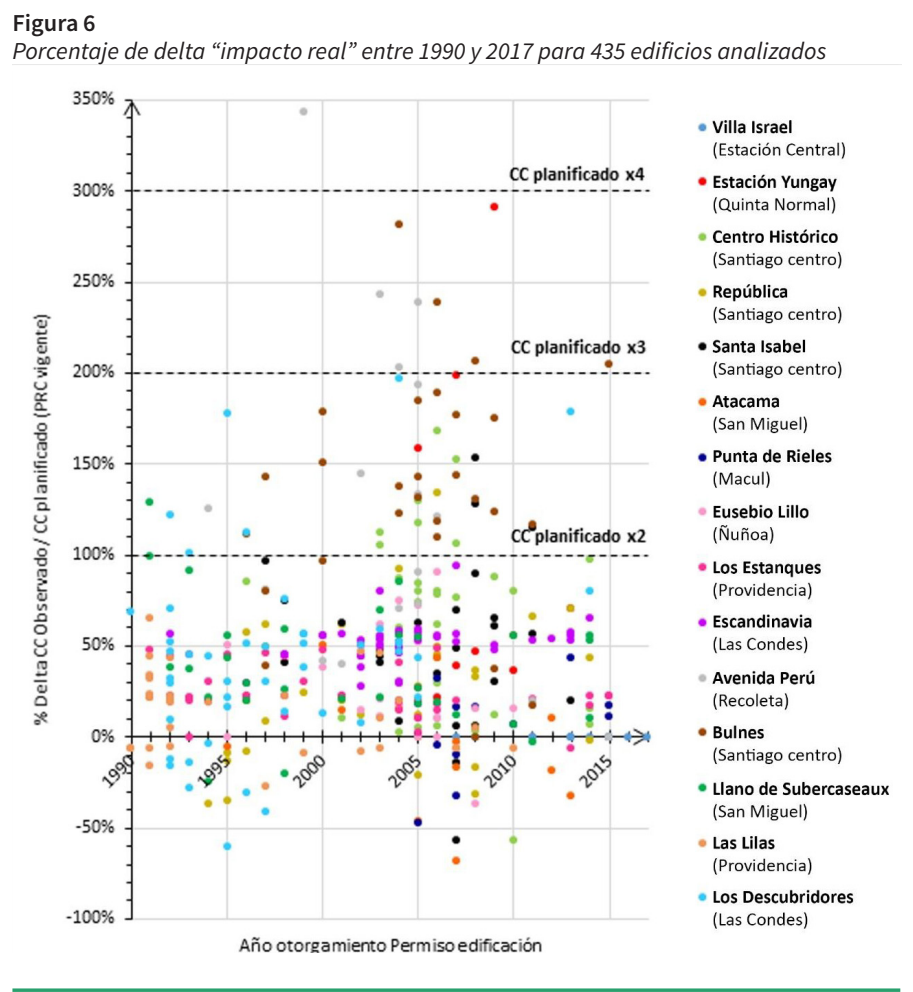

Figura 7

Imágenes satelitales de los ejemplos en barrios Bulnes y Atacama

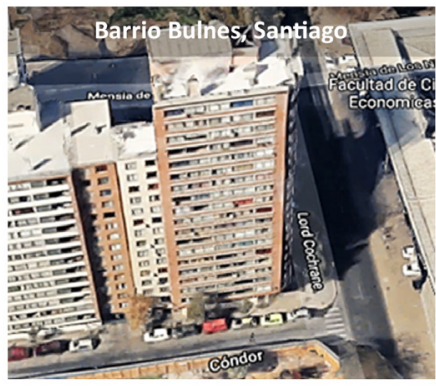

C.C. observado: 13,74

C.C. planificado: 3,60

Delta: $\mathbf{+ 2 8 1 , 6 \%}$

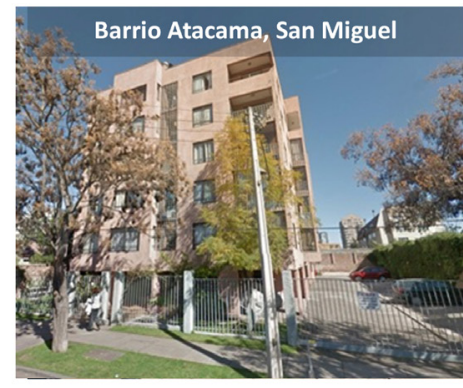

C.C. observado: 1,12

C.C. planificado: 3,25

Delta: $-68,7 \%$
Un 12,2 \% de los edificios analizados presentan deltas negativos, lo que refleja mayor permisividad normativa posterior al desarrollo del edificio. Por otro lado, en un $61,9 \%$ de los casos este delta es positivo, y el C.C. observado supera el planificado en hasta 2 puntos, como ocurre en los barrios Bulnes y Avenida Perú. En un $22 \%$ de los casos el delta es positivo y el C.C. observado supera el planificado en más de 2 puntos. En los últimos dos escenarios mencionados, estas diferencias responden al uso de beneficios de constructibilidad propios de las normas de excepción, o bien a modificaciones del PRC que disminuyen el C.C después de aprobado el permiso de edificación.

En el ejemplo presentado para el barrio Bulnes (Figura 7), el C.C. planificado corresponde a 3,6 y el observado a 13,74. Por el contrario, en el caso del Barrio Atacama, el C.C. observado corresponde a 1,02 y el C.C. planificado a 3,25. Esta diferencia expresa que la norma de constructibilidad es más permisiva que el desarrollo inmobiliario anterior a su aprobación. 
Tabla 3

Presencia de los beneficios de excepción por fusión predial y conjunto armónico en los barrios analizados

\begin{tabular}{lll}
\hline Barrio & $\begin{array}{l}\text { Porcentaje de } \\
\text { edificios con } \\
\text { fusión predial }\end{array}$ & $\begin{array}{l}\text { Porcentaje } \\
\text { de edificios } \\
\text { con conjunto } \\
\text { armónico }\end{array}$ \\
\hline Avenida Perú & $64,70 \%$ & $5,90 \%$ \\
\hline Las Lilas & $51,20 \%$ & $0,00 \%$ \\
\hline Escandinavia & $97,80 \%$ & $4,30 \%$ \\
\hline Los Descubridores & $70,20 \%$ & $1,80 \%$ \\
\hline Eusebio Lillo & $87,00 \%$ & $0,00 \%$ \\
\hline República & $78,80 \%$ & $0,00 \%$ \\
\hline Los Estanques & $38,30 \%$ & $0,00 \%$ \\
\hline $\begin{array}{l}\text { Llano de } \\
\text { Subercaseaux }\end{array}$ & $35,50 \%$ & $0,00 \%$ \\
\hline Atacama & $73,30 \%$ & $0,00 \%$ \\
\hline Punta de Rieles & $38,50 \%$ & $0,00 \%$ \\
\hline Bulnes & $67,90 \%$ & $0,00 \%$ \\
\hline Centro Histórico & $70,60 \%$ & $0,00 \%$ \\
\hline Promedio edificios $\%$ & 0,691 & 0 \\
\hline analizados $(n=144)$ & & \\
\hline
\end{tabular}

\section{Delta fusión predial y/o conjunto armónico: Incremento de hasta un 30 $\%$ de constructibilidad por sobre el PRC local}

El delta “fusión predial y/o conjunto armónico" expresa la diferencia entre el C.C. proyectado y el C.C. permitido por el PRC, ambos consignados en el permiso de edificación. El C.C. proyectado no necesariamente corresponde al C.C. observado, porque, como señalamos anteriormente, el C.C. proyectado solo considera la superficie útil o vendible. Este delta solo expresa la utilización del beneficio por premio por fusión predial y/o por conjunto armónico.

El promedio observado para este delta es de 22,1 \%, el cual corresponde a un C.C. neto de 0,4 , equivalente a la diferencia entre el C.C. proyectado promedio $(2,9)$ y el C.C. permitido promedio $(2,5)$ (Tabla 1$)$. Este valor se explica por la aplicación del premio por fusión predial en un 69,1\% de los casos y, en una proporción muy menor por la figura del conjunto armónico, que se identifica en apenas un $1 \%$ de los casos. La variabilidad del desempeño de este delta es menor que la del delta impacto real (Figura 4).
Mientras el premio por fusión predial aporta al edificio, en promedio, una superficie de $1.200 \mathrm{~m}^{2}$ construidos adicionales a los permitidos, solo se identificaron cuatro casos que utilizan el premio por conjunto armónico: dos casos en Escandinavia, uno en Los Descubridores y uno en Avenida Perú. Los tres primeros además aplicaron el premio por fusión predial, aumentando entre un $43 \%$ a $56 \%$ el C.C. permitido, lo que se traduce en cerca de $5.200 \mathrm{~m}^{2}$ adicionales por proyecto. El caso en Avenida Perú, que goza únicamente del beneficio de conjunto armónico (30\%), presenta una diferencia de $2.900 \mathrm{~m}^{2}$ adicionales al C.C. permitido por el PRC.

En términos de superficie edificada, este delta alcanza un promedio de $13.153 \mathrm{~m}^{2}$ en la escala barrial. Los barrios con deltas fusión predial o conjunto armónico más altos corresponden a Escandinavia (29\%), Los Descubridores (27,5 \%), Avenida Perú (34,8 \%) y Las Lilas (30\%), principalmente por la extendida utilización de del beneficio de fusión predial (Tabla 3). Una estructura predial relativamente homogénea, así como normas de superficie predial mínima e incentivos en el PRC propician la densificación del barrio con operaciones de fusión predial.

Por el contrario, los Barrios Bulnes y Centro Histórico presenta deltas negativos de $-0,5 \%$ y $-7,5 \%$, respectivamente, principalmente porque el PRC de Santiago define para estas zonas una altura máxima que limita el crecimiento de los proyectos de densificación, no alcanzando toda la constructibilidad permitida.

\section{Delta superficie DFL2: constructibilidad adicional no expresada en el permiso de edificación}

El delta superficie DFL2 corresponde a la diferencia entre el C.C. observado y el C.C. proyectado en el permiso de edificación (Figura 2). Expresa la superficie común (no vendible) de los edificios, que en un $65 \%$ de los casos no se encuentra considerada dentro del cálculo de la constructibilidad proyectada consignada en el permiso de edificación. Dado que solo es posible considerar hasta un $20 \%$ como superficie común, este delta presenta 
valores más bajos y con menor variabilidad que los otros deltas analizados.

El delta superficie DFL2 alcanza un promedio de $17 \%$, el cual corresponde a un C.C. neto de 0,6 , equivalente a la diferencia entre el C.C. promedio observado $(4,5)$ y el C.C. proyectado $(3,9)$ (Tabla 1). En términos de superficie edificada, el delta superficie DFL2 alcanza un total de $197.302 \mathrm{~m}^{2}$ edificados totales, con un valor promedio de $1.370 \mathrm{~m}^{2}$ por proyecto, y $13.153 \mathrm{~m}^{2}$ en la escala barrial. Los barrios con un delta superficie DFL2 más alto (cercano al $20 \%$ ) corresponden a Avenida Perú, Punta de Rieles y Eusebio Lillo. Aquellos con un delta más bajo corresponden a los barrios Bulnes, Las Lilas y Estación Yungay, los cuales presentan deltas menores a $10 \%$.

\section{Delta modificaciones PRC: Debilidad de los instrumentos de planificación frente al mercado inmobiliario}

Finalmente, el delta "modificaciones PRC" expresa la diferencia entre el C.C. proyectado (consignado en el permiso de edificación) y el C.C. actualmente normado en el PRC (Figura 2). Permite visualizar modificaciones a los PRC en el período entre la edificación de la torre residencial y la última modificación al PRC. Todo valor positivo refleja una tendencia del PRC a limitar la constructibilidad, y todo valor negativo indica una modificación en pos de mayor permisividad normativa. Puede evidenciar la debilidad de los PRC para planificar oportunamente la densificación residencial intensiva en contextos de rápida transformación.

Este delta presenta un promedio de $28,6 \%$, el cual corresponde a un C.C. neto de 0,7 , equivalente a la diferencia entre el C.C. promedio proyectado $(3,8)$ y el C.C. actualmente planificado $(3,1)$ (Tabla 1). Un $70 \%$ de los edificios presenta valores positivos, expresando el uso de mayor constructibilidad atribuible a los beneficios de las normas de excepción, o la disminución del C.C. en los respectivos $\mathrm{PRC}$, desde el otorgamiento del permiso de edificación. Un 7,2 \% de los casos presenta deltas iguales a cero, esto es, el C.C. proyectado coincide con el planificado actualmente. Un $23 \%$ de los casos presenta deltas negativos, lo que indica que no se aplicó la constructibilidad máxima (limitación por alturas, rasante u otras normas), o bien, se incrementó el C.C. en forma posterior a la aprobación del permiso de edificación. Esto datos permiten corroborar que en la mayor parte de los casos la limitación de las condiciones de constructibilidad ha llegado con posterioridad al desarrollo de proyectos de densificación en altura en los barrios.

Avenida Perú, República y Punta de Rieles presentan deltas cercanos a $0 \%$ y de valor negativo, lo que expresa pocas modificaciones a los PRC en términos de C.C. En barrios como Atacama, Punta de Rieles o Las Lilas, estas diferencias son menores, producto de un bajo nivel de modificación del PRC. En República, un ligero incremento en este delta responde a que el C.C. permitido se modificó de 2,2 en 1989, a 3,6 y 2,8 en 2011.

Aunque este delta presenta un desempeño relativamente similar al delta impacto real, es posible destacar diferencias en barrios como Avenida Perú, Escandinavia, Eusebio Lillo, Punta de Rieles, Estación Yungay y República. En Estación Yungay la modificación al PRC (2016) limita las condiciones para la densificación intensiva, desde una norma que restringía la densificación solo con altura máxima según rasante de $70^{\circ}$, a limitar la capacidad edificada mediante un C.C. de 1,2 y altura máxima de cinco pisos. Así, el C.C. proyectado duplica el C.C. actualmente planificado.

Los PRC de Santiago y Recoleta han tendido a normar constructibilidades para áreas residenciales en torno a 7,2 y 2,4 con alturas entre 13 y 38 pisos, llegando tardíamente a la conducción de este proceso de transformación urbana, cuando el tejido ya se encuentra hiperdensificado. En consecuencia, con pocas posibilidades de continuar el proceso de renovación urbana bajo nuevas reglas del juego que promueven una densificación más equilibrada.

En Bulnes, por ejemplo, donde no existían restricciones de constructibilidad y altura máxima, el 2011 se introduce un C.C. de 3,6 con altura máxima de 22,5 metros. La Figura 8 ejemplifica el "retraso" de la norma urbanística respecto de las rápidas dinámicas de verticalización 


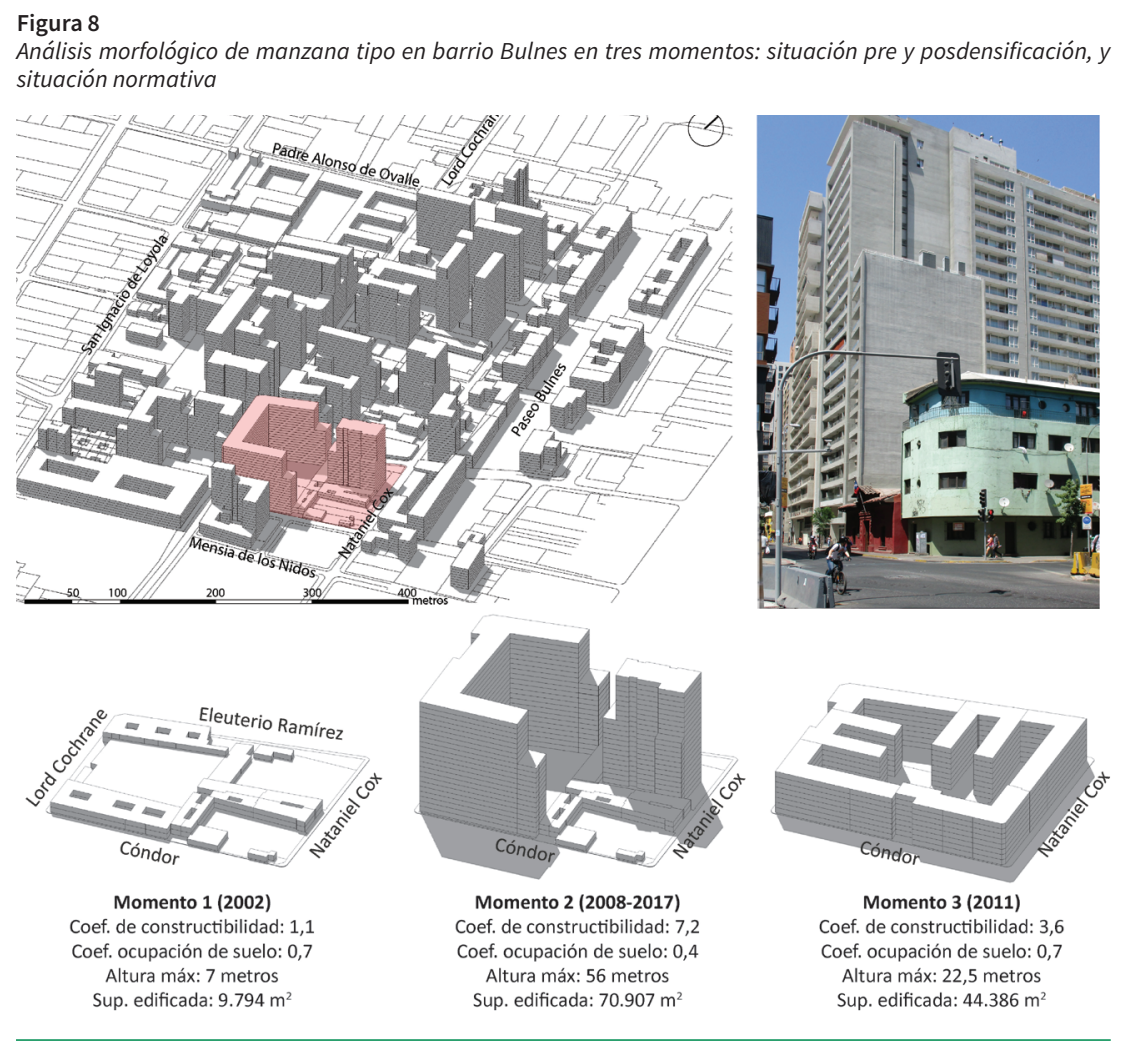

de este barrio, donde los permisos de edificación se aprobaron entre los años 1996 y 2015 (anteproyectos ingresados con anterioridad). En el primer momento se presenta el tejido urbano preexistente al proceso de densificación residencial. En el segundo momento se presenta la manzana densificada entre los años 2008 y 2017. El tercer momento representa la cabida teórica de la norma urbanística aprobada en 2011. Cabe señalar que el segundo momento alcanza una superficie construida que es un $37 \%$ inferior a la del momento tres, ya que en este último caso dos edificios concentran el potencial densificatorio de la manzana con torres de más de 20 pisos. Observamos que la norma urbanística distribuye la edificabilidad en la manzana con menor altura y mayor ocupación de suelo, quintuplicando la constructibilidad del terreno. En otras palabras, la norma urbanística prefigura una transformación más compacta y homogénea de la manzana, en mayor diálogo y armonía con el contexto urbano preexistente.

Por su parte, algunos PRC han restringido el uso de los beneficios de excepción por fusión predial y/o conjunto armónico, como es el caso de Santiago y Providencia. Sin embargo, el Dictamen $N^{\circ} 4.373$ (Dictamen CGR, de 19 de julio de 2019, sobre planes reguladores) precisa que la hegemonía de los PRC se limita a los cuerpos normativos fijados en la LGUC y la OGUC y que, por lo tanto, no puede intervenir respecto del uso de dichos beneficios a través de restricciones que impidan la aplicación de dichos premios.

Constatamos también que el delta modificaciones PRC presenta mayores brechas en la escala del edificio que en la barrial. La Figura 9 ilustra dos casos disímiles. En el caso del barrio Los Descubridores, la constructibilidad proyectada es un $60,1 \%$ menor a la permitida actualmente por el PRC de Las Condes. Esta diferencia expresa que la norma de constructibilidad es más permisiva que el desarrollo inmobiliario anterior a su aprobación, lo cual hace sentido al considerar que el edificio fue aprobado en 1977, cuando aún no entraba en vigencia el actual PRC de Las Condes. Esta mayor permisividad normativa posdensificación también se reconoce en los barrios Llano de Subercaseaux, Eusebio Lillo, Los Descubridores, Punta de Rieles y Centro Histórico.

Por el contrario, en el caso seleccionado para el Barrio Bulnes, observamos que el C.C. proyectado alcanzado por el edificio supera en un $291,9 \%$ al C.C. normado por el PRC. Esto se condice con el antecedente de la 
Figura 9

Imágenes satelitales de los ejemplos en Los Descubridores y Bulnes

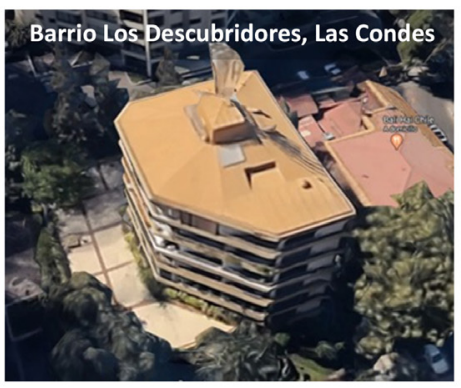

C.C. proyectado: 1,04 C.C. planificado: 2,60 Delta: $-60,13 \%$

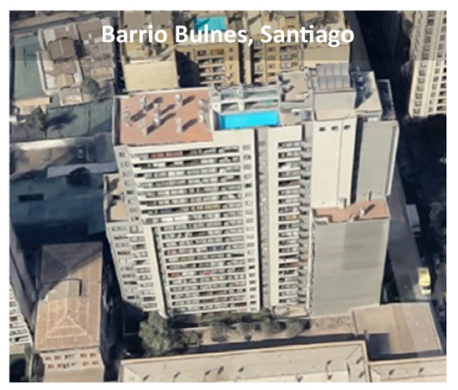

C.C. proyectado; 14,11

C.C. planificado: 3,60

Delta: $+291,94 \%$ modificación de 2011, que limita la edificabilidad de edificios en altura.

\section{Conclusiones}

Este trabajo ha permitido evidenciar, desde un punto de vista cuantitativo, el impacto de las normas de fusión predial, conjunto armónico y DFL2 en la constructibilidad del proyecto residencial en altura en barrios verticalizados del AMS. Los deltas aquí propuestos constituyen indicadores que permiten evaluar en qué medida, normas de nivel nacional y carácter general inciden en el potencial edificatorio del proyecto residencial en altura. A su vez, contribuyen a explicar la debilidad de la planificación urbana local frente a disposiciones centralizadas, originadas hace más de cinco décadas como respuesta a contextos político-económicos sumamente diferentes a los actuales, tales como el repoblamiento de áreas centrales y las políticas habitacionales orientadas a disminuir el déficit habitacional (Schlack y Vicuña, 2011).

La Ley $\mathrm{N}^{\circ} 20.958$ de Aportes al Espacio Público, del Ministerio de Vivienda y Urbanismo y el Ministerio de Transportes y Telecomunicaciones, ha restringido la aplicación del premio por fusión predial y el conjunto armónico en zonas donde los PRC establezcan incentivos para la integración social y la sustentabilidad. Esta disposición permitirá fortalecer la capacidad de los municipios para conducir los procesos de densificación mediante beneficios y condiciones integradas a los PRC y a la definición de una imagen objetivo y visión de ciudad consensuada.

La amplia utilización del premio por fusión predial y del DFL2 (63 \% y 99,8 \% de la muestra, respectivamente) indica que cabría evaluar la eliminación o al menos la revisión de estos beneficios, considerando también los altos niveles de inversión y dinamismo del mercado inmobiliario en las principales ciudades del país.

En efecto, los deltas de superficie construida, agregados en la escala barrial, dan cuenta de la relevancia de esta problemática. Los rápidos procesos de verticalización han superado la capacidad de gran parte de los municipios para responder oportunamente con normas urbanísticas que permitan adelantarse a la utilización de estos beneficios, y cautelar así condiciones razonables de habitabilidad urbana y residencial.

Cabría suponer que la baja utilización de la norma de conjunto armónico (1\% de la muestra), podría responder a que las condiciones normativas de los barrios analizados son favorables a la verticalización, o que las condiciones que exige este beneficio son más recurrentes en otras condiciones urbanas. En el contexto actual de rápida transformación de las áreas metropolitanas chilenas, altos niveles de inversión en vivienda en altura, e importantes demandas por parte de la ciudadanía por procesos de planificación urbana participativos, es necesario indagar en la incidencia de la norma de conjunto armónico y sus efectos en los barrios donde se emplazan dichas operaciones.

Las “normas de excepción” a la planificación urbana, como las hemos denominado, no solo inciden en la producción de la ciudad verticalizada. En los procesos de expansión en la periferia y el periurbano, es posible detectar normas generales que, por ejemplo, permiten urbanizar fuera del límite urbano establecido por el instrumento de planificación. La literatura define las prácticas de excepción como reglas y procedimientos especiales en situaciones específicas (Baptista, 2013; Marrero-Guillamón, 2011,). Sin embargo, el caso chileno da cuenta de una práctica 
extendida y generalizada, que ilustra lo que Watson (2009) ha denominado "conflictos de racionalidades" dentro del aparato estatal, el cual promueve activamente la planificación urbana local, al tiempo que soslaya la persistencia de este tipo de dispositivos legales que la debilitan. Las razones y circunstancias tras la persistencia de dichas excepciones debieran ser materia de atención analítica y revisión crítica por parte de la academia.

\section{Referencias bibliográficas}

Alcaíno, J. (2008). Efectos de las normas de excepción sobre la planificación urbana comunaly sobre los conflictos urbanos [Tesis de Magíster]. Pontificia Universidad Católica de Chile, Santiago, Chile.

Asencio, S. (7 de abril de 2019). Justicia acoge demanda de U. de Chile contra inmobiliaria responsable de megaedificios en Bellavista. Biobio Chile. https://www.biobiochile. cl/noticias/nacional/region-metropolitana/2019/04/07/ justicia-acoge-demanda-de-u-de-chile-contra-inmobiliararesponsable-de-megaedificios-en-pio-nono.shtml

Baptista, I. (2009). Regimes of exception in urban planning and governance: the case of the Polis program, Portugal [Tesis de doctorado].Universidad deCalifornia, Berkeley, Estados Unidos.

Baptista, I. (2013). Practices of Exception in Urban Governance: Reconfiguring Power Inside the State. Urban Studies, 50(1) 39-54. https://doi.org/10.1177/0042098012453858

Boyer, R., (1989). La teoría de la regulación: un análisis crítico. SECYT; CONICET; Editorial Hvmanitas.

Consejo Nacional de Desarrollo Urbano. (2016). Sistema de planificación urbana integrada. Propuestas para la implementación de la Política Nacional de Desarrollo Urbano. https://cndu.gob.cl/wp-content/uploads/2016/04/ Planificaci\%C3\%B3n-Urbana-Integrada_LEB-CNDU_abril-2016.pdf

Consejo Nacional de Desarrollo Urbano. (2019). Propuestas para una densificación equilibrada en las ciudades chilenas. https://cndu.gob.cl/download/propuestas-para-unadensificacion-equilibrada-en-las-ciudades-chilenas/
Contreras, Y. (2011). La recuperación urbana y residencial del centro de Santiago: Nuevos habitantes, cambios socioespaciales significativos. EURE (Santiago), 37(112), 89-113. https://dx.doi.org/10.4067/S025071612011000300005

DDU 110, 2002 / Circular Ordinaria Nº 300 / [División de Desarrollo Urbano, Ministerio de Vivienda y Urbanismo] / Artículos 5.1.11. y 6.1.5. de la Ordenanza General de Urbanismo y Construcciones, SUPERFICIE EDIFICADA, CALCULO DE SUPERFICIE / DDU, 14.08.2002. 14 de agosto de 2002.

Decreto $\mathrm{N}^{\circ} 2$ de 1959 [con fuerza de ley]. FIJA TEXTO DEFINITIVO DEL DFL 2, DE 1959, SOBRE PLAN HABITACIONAL. 07 de julio de 1959. 31 de julio de 1959.

De Oliveira, P., De Oliveira, P., Mendes, W., De Oliveira, M. y Soraggi, M. (2015). Verticalização consciente: edificar integrando ao meio urbano. REINPEC-Revista Interdisciplinar Pensamento Científico, 1(1), 12-27. https:// doi.org/10.20951/2446-6778/v1n1a2

Dictamen N 4.373 [Contraloría General de la República de Chile]. Sobre "Plan Regulador Comunal de Santiago (PRC)". 19 de julio de 2019.

Diez, F. (1996). Buenos Aires y algunas constantes en las transformaciones urbanas. Fundación Editorial Belgrano.

Fainstein, S. S., \& Fainstein, N. I. (1971). City planning and political values. Urban Affairs Quarterly, 6(3), 341-362.

Farías, I., \& Flores, P. (2015). A different state of exception: Governing urban reconstruction in post-27F Chile. Urban Studies, 54(5) 1108-1125. https://doi. org/10.1177/0042098015620357

Fedele, J. y Martínez, I. (2015). Verticalización y desarrollo inmobiliario del núcleo urbano central de Santa Fe: cambios morfológicos, conflictos urbanos y regulaciones edilicias en la recuperación poscrisis 2001. Cuaderno Urbano, 18(18), 65-88. https://doi.org/10.30972/ crn. 1818265 
Gerencia de Estudios, TOCTOC.com. (2020). El desarrollo urbano atomizado en el Gran Santiago: Oportunidades en Cerrillos, Apunte de Ciudad N² (Documento inédito).

Greene, M., Link, F., Mora, R. y Figueroa, C. (2014). From the Home to the Neighborhood. ARQ (Santiago), (86), 78-87. https://dx.doi.org/10.4067/S0717-69962014000100012

Harvey, D. (1998). La condición de la posmodernidad. Amorrortu.

Hidalgo, R. (2010). Los centros históricos y el desarrollo inmobiliario: las contradicciones de un negocio exitoso en Santiago de Chile. Scripta Nova. Revista Electrónica de Geografía y Ciencias Sociales, 14(331), 85. http://www. ub.es/geocrit/sn/sn-331/sn-331-85.htm

Ilustre Municipalidad de Providencia (2007). Memoria explicativa Plan Regulador Comunal de 2007. https://providencia.cl/ provi/site/docs/20191112/20191112162159/prcp_2007_ memoria_explicativa.pdf

Innocenti, D., Fulgueiras, M. y Mora, P. (2014). ¿Densificación como vía para conciliar negocio inmobiliario e integración social? El caso de la comuna de Santiago de Chile. Pontificia Universidad Católica de Chile.

Inzulza-Contardo, J. (2012). ¿Latino-gentrificación? Focusing on Physical and Socioeconomic Patterns of Change in Latin American Inner Cities. Urban Studies, 49(10), 2085-2017. https://doi.org/10.1177/0042098011423425

Kostof, S. (1991). The City Shaped. Urban Patterns and Meanings Through History. Bulfinch Press.

Laurian, L., Day, M., Berke, P., Ericksen, N., Backhurst, M., Crawford, J., \& Dixon, J. (2004). Evaluating Plan Implementation: A Conformance-Based Methodology. Journal of the American Planning Association, 70(4), 471-480. https://doi.org/10.1080/01944360408976395

Ley N²0.958 de 2016. Aportes al Espacio Público. 15 de octubre de 2016. Diario Oficial de la República de Chile $N^{\circ} 41.584$.
Ley N²1.202 de 2020. Ley General de Urbanismo y Construcciones. 23 de enero de 2020. Diario Oficial de la República de Chile $\mathrm{N}^{\circ} 42.560$.

Livert, F., \& Gainza, X. (2014). Understanding density in an uneven city, Santiago de Chile: implications for social and environmental sustainability. Sustainability, 6(9), 5876-5897.

López-Morales, E. (2013). Gentrificación en Chile: aportes conceptuales y evidencias para una discusión necesaria. Revista de Geografía Norte Grande, (56), 31-52. https:// dx.doi.org/10.4067/S0718-34022013000300003

López-Morales, E., Arriagada-Luco, C., Gasic-Klett, I. y MezaCorvalán, D. (2015). Efectos de la renovación urbana sobre la calidad de vida y perspectivas de relocalización residencial de habitantes centrales y pericentrales del Área Metropolitana del Gran Santiago. EURE (Santiago), 41(124), 45-67. https://dx.doi.org/10.4067/S0250$\underline{71612015000400003}$

López-Morales, E., Gasic Klett, I. y Meza Corvalán, D. (2012). Urbanismo pro-empresarial en Chile: políticas y planificación de la producción residencial en altura en el pericentro del Gran Santiago. Revista INVI, 27(76), 75-114. https://dx.doi.org/10.4067/S0718-83582012000300003

Lozano, E. (1990). Density in communities, or the most important factor in building urbanity. En E. Lozano (Ed.), Community design and the culture of cities: The crossroad and the wall (pp. 157-183). MIT Press.

Machado, J. R., \& Méndes, C. M. (2003). O processo de verticalização do centro de Maringá-PR, Brasil. Investigaciones geográficas, (52), 53-71.

Marrero-Guillamón, I. (2011). London 2012: espacio de excepción. Urbe. Revista Brasileira de Gestão Urbana (Brazilian Journal of Urban Management), 3(2), 179190. https://www2.pucpr.br/reol/pb/index.php/ urbe?dd1 $=5725 \& d d 99=$ view $\& d d 98=$ pb 
Mora, P., Polanco, M. y Innocentti, D. (2013). Hacia un modelo sustentable de densificación: la mirada desde los ciudadanos. Pontificia Universidad Católica de Chile.

Ordenanza General de Urbanismo y Construcciones de 2020 [Ministerio de Vivienda y Urbanismo de Chile]. 13 de junio de 2020.

Organización de las Naciones Unidas. (2016). Nueva Agenda Urbana aprobada en la Conferencia de las Naciones Unidas sobre la Vivienda y el Desarrollo Urbano Sostenible (Hábitat III). Edición del autor. https://uploads.habitat3. org/hb3/NUA-Spanish.pdf

Orellana, A., Vicuña, M. y Moris, R. (2017). Planificación urbana y calidad de vida: Aproximación al estado de la planificación local en Chile. Cuadernos de Vivienda y Urbanismo, 10(19), 88-109. https://doi.org/10.11144/ Javeriana.cvu10-19.pucv

Rojas, L. (2017). Ciudad vertical: la "nueva forma" de la precariedad habitacional comuna de Estación Central, Santiago de Chile. Revista 180, (39). http://www. revista180.udp.cl/index.php/revista180/article/ view/365/318

Sabaté, J. (1994). El proyecto de la calle sin nombre: los reglamentos urbanos de la edificación. En E. Serra Riera, E. Gómez y M. Jiménez (Eds.), 12 tesis en relació al procés de transformació de l'eixample Cerdà de Barcelona (pp. 96-103). Edicions ETSAB-UPC.

Sáez, S. (10 de febrero de 2021). Conflicto entre desarrolladores y vecinos está en la justicia ambiental. La nueva estrategia de Ana Lya Uriarte para judicializar proyecto Portezuelo en Lo Curro. Litoral Press. https://www. litoralpress.cl/sitio/Prensa_Texto?LPKey=idfrmo0. Fz.Jz5s.X.C.Gpdyks9.X.Mu5d5ip.Ni.D.Mbdv.Hw25. Ug. $\%$ C $3 \% 96$

Sanhueza, M. y Peña-Cortés, F. (2010). Planes reguladores comunales en la Región del Bío-Bío: Estado y situación actual. Urbano, 13(22), 32-42. http://revistas.ubiobio. cl/index.php/RU/article/view/304
Schlack, E. y Vicuña, M. (2011). Componentes normativas de alta incidencia en la nueva morfología del Santiago Metropolitano. EURE(Santiago), 37(111), 131-166. https:// doi.org/10.4067/s0250-71612011000200006

Scussel, M., \& Sattler, M. (2010). Cities in (trans) formation: The impact of verticalization and densification on the quality of residential space. Ambiente Construido, 10(3), 137-150. https://doi.org/10.1590/S1678-86212010000300009

Señoret, A. y Link, F. (2019). Densidad urbana, forma y sociabilidad en la ciudad neoliberal: el caso del barrio Santa Isabel en Santiago de Chile. Revista de Urbanismo, (41). https://doi.org/10.5354/0717-5051.2019.52862

Swyngedouw, E., Moulaert, F., \& Rodriguez, A. (2002). Neoliberal Urbanization in Europe: Large-Scale Urban Development Projects and the New Urban Policy. Antipode, 34(3), 542-577. https://doi.org/10.1111/1467-8330.00254

Tella, G., Cañellas, E., Muñoz, M. y Natale, D. (2011). Importando la torre al barrio. Procesos de densificación intensiva en la periferia metropolitana de Buenos Aires. Revista Iberoamericana de Estudios Municipales, 2(3), 175-199. https://doi.org/10.32457/riem.vi3.433

Vergara-Perucich, J. F., \& Aguirre-Nuñez, C. (2020). Housing Prices in Unregulated Markets: Study on Verticalised Dwellings in Santiago de Chile. Buildings, 10(1), 6. https:// doi.org/10.3390/buildings10010006

Vergara Vidal, J. (2017). Verticalización. La edificación en altura en la Región Metropolitana de Santiago (1990-2014). Revista INVI, 32(90), 9-49. https://dx.doi.org/10.4067/ S0718-83582017000200009

Vialard, A. (2012). Measures of the fit between street network, urban blocks and building footprints [Tesis de doctorado]. Georgia Institute of Technology, Atlanta, GA., Estados Unidos.

Vicuña, M. (2015). Las formas de la densidad residencial. El caso del Gran Santiago [Tesis de doctorado]. Pontificia Universidad Católica de Chile, Santiago, Chile. 
Vicuña, M. (2017). Impacto de la densificación residencial intensiva en la segmentación del tejido urbano de Santiago: un enfoque cuantitativo. Revista 180, (40).

Vicuña, M. (2020). Densidad y sus efectos en la transformación espacial de la ciudad contemporánea: cinco tipologías para interpretar la densificación residencial intensiva en el Área Metropolitana de Santiago. Revista 180, (45). https://doi.org/10.32995/rev180.num-45.(2020).art-659

Wai-Chung Lai, L. (2002). Libertarians on the road to town planning: A note on the views of Robert Mundell, Karl Popper, Friedrich Hayek, Robert Nozick, Milton Friedman and Ronald Coase towards pollution. Town Planning Review, 73(3), 289-310. https://doi.org/10.3828/tpr.73.3.4

Watson, V. (2009). Seeing from the South: Refocusing urban planning on the globe's central urban issues. Urban Studies, 46(11), 2259-2275. https://doi. org/10.1177/0042098009342598 